\title{
Comparisons of Feed-Forward and Multiple-Feedback Sigma-Delta Modulators for MEMS Accelerometers
}

\author{
Zhang Meimei , Quan Haiyang and Zhang Fuqiang \\ Beijing Microelectronics Technology Institute, 100076 Beijing, China
}

\begin{abstract}
This paper investigates two different architectures of a $5^{\text {th }}$ order electro-mechanical sigma-delta modulator: a feed-forward (FF) architecture and a multiple-feedback (MF) architecture. And a comparison was performed in terms of stability and noise shaping ability, sensitivities to parameter variances due to fabrication tolerances and loop gain, and nonlinearity in feedback force. Both architectures were modeled in Simulink and investigated at system level. The results show that: a) both architectures are stable and achieve the similar noise floor level of -170dB within $250 \mathrm{~Hz}$ in the ideal condition; b) both architectures have good ability in fabrication tolerance; c) the performance of the MF architecture will degrade heavily with the loop gain decreasing and become unstable if the loop gain beyond one optimal value, while the FF architecture is insensitive; d) the FF architecture controls the proof mass well and achieves better SNDR, whereas the MF has a $56 \mathrm{~dB}$ degradation in consideration of nonlinearity in feedback force.
\end{abstract}

\section{Introduction}

MEMS (micro-electro-mechanical systems) capacitive sensing elements are commonly embedded in a closed loop, force feedback system to realize increased dynamic range, better linearity and wider bandwidth [1]. In particular, incorporated in a sigma delta modulator (SDM), the system can output direct digital signal, and avoid potential electro-static instability [2]. Earlier works showed that only using the MEMS sensing element as the loop filter, forming a second order electro-mechanical sigma delta modulator (EMSDM), suffers from relatively high quantization noise, idle tones and dead-zones [3]. To address these weaknesses, several research groups have designed high order EMSDMs, which cascading the sensing element with an electronic filter. The architecture of EMSDMs can be developed from conventional electronic SDMs, which can be sorted into single loop and multi stage noise shaping (MASH). Although MASH architectures have the considerable advantage of inherent stability and large input range, the severe drawback of the MASH is its sensitivity to component and parameter tolerances [4]. In contrast, the single loop architectures have been applied successfully to capacitive MEMS accelerometers [5, 6, 7, 8]. In this paper, the author discussed two typical architectures of the single loop, called the feed-forward (FF) and the multiple-feedback (MF), respectively. Simulation results showed that the FF has better performance in EMSDMs.

This paper is organized as follows: Section 2 describes the system level models of two architectures in general; Section 3 compares the FF and MF in terms of sensitivity to parameter variations and the loop gain, and nonlinearity in the feedback force; in Section 4 conclusions are drawn.

\section{System level modeling}

The Simulink models of the FF and MF are illustrated in Figure 1 and Figure 2, respectively. Traditionally, an EMSDM accelerometer system consists of the MEMS capacitive sensing element and the electrical interface circuit [9]. The sensing element, which is modeled as a mass-damping-spring system with a second order transfer function, converts acceleration into the displacement of the proof mass, which is reflected by the change of differential capacitances. The electrical interface circuit often includes the following blocks: the read-out circuit that outputs a voltage proportional to the displacement by measuring capacitor change; a phase compensator providing phase-lead compensation to stabilize the system (especially when the sensing element is under damped); an electronic loop filter that shapes quantization noise to high frequencies by oversampling; a quantizer outputting a digital bitstream, which decides the direction of the feedback electrostatic force to keep the displacement of the proof mass small.

To analyze both architectures mathematically, the quantizer is usually assumed as a simple gain $(\mathrm{Kq})$ with additive white noise [10]. For small mass deflections, the pickoff and feedback circuits can also be modeled simply as gain constants $\mathrm{Kxv}$ and $\mathrm{Kfb}$, respectively. Based on the above assumptions, the quantization noise transfer functions for the FF and the MF are given by: 


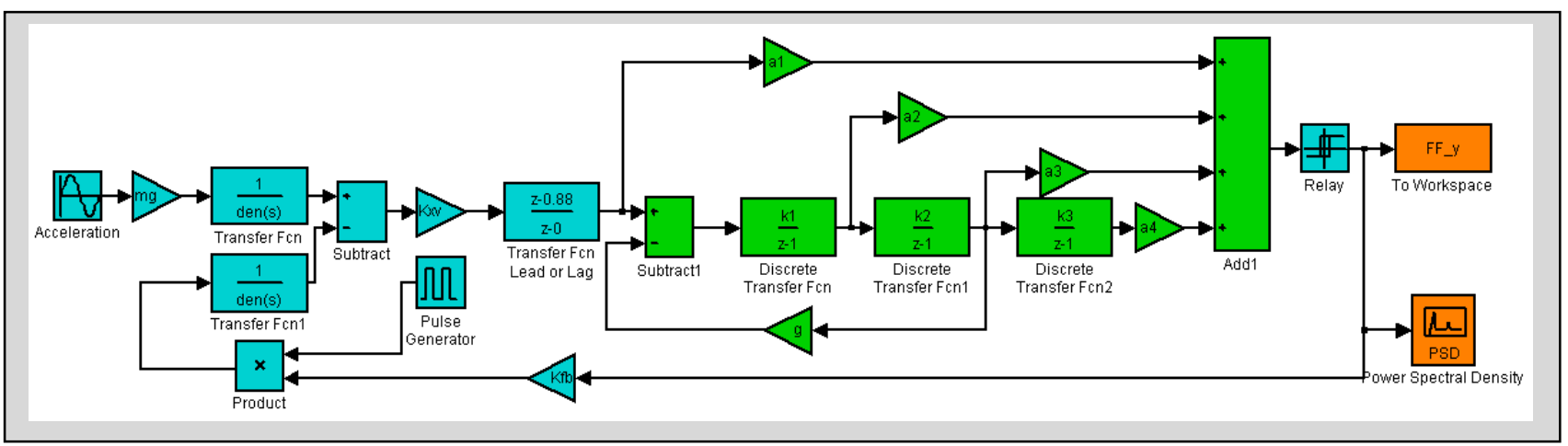

Figure 1. Simulink model of a FF $5^{\text {th }}$ order EMSDM.

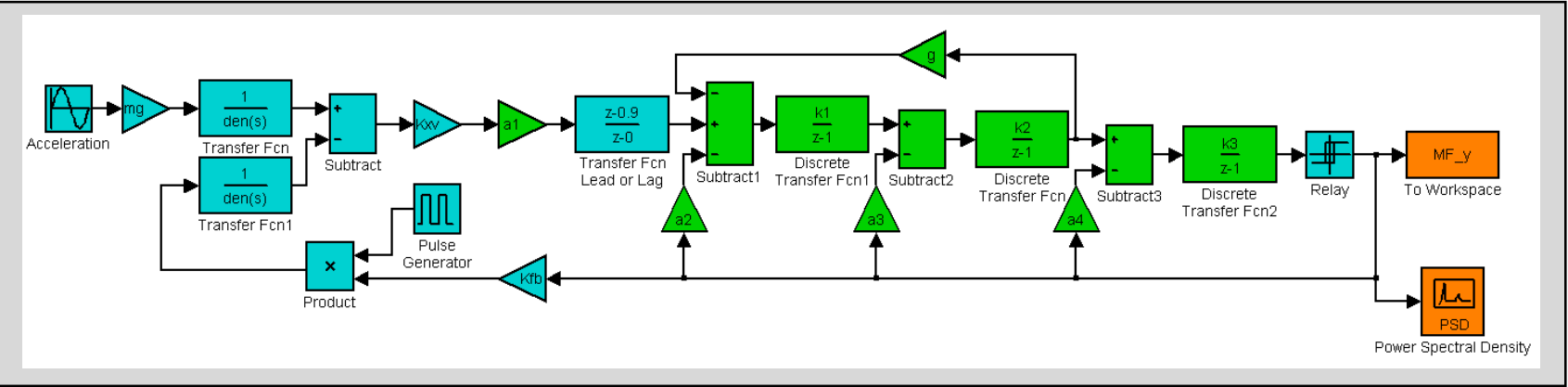

Figure 2. Simulink model of a MF $5^{\text {th }}$ order EMSDM

$Q N T F_{-} F F=\frac{1}{1+K_{q} K_{f b} K_{x v} H_{m}(z) H_{c}(z) H_{F F_{-} e}(z)}$

$Q N T F_{-} M F=\frac{1}{1+K_{q} H_{M F_{-} e 1}(z)+K_{q} K_{f b} K_{x v} H_{m}(z) H_{c}(z) H_{M F_{-} e 2}(z)}$

where $H_{m}(z)$ is the transfer function of the sensing element in discrete-time domain [11]:

$$
H_{m}(z)=\frac{K_{m}(z+\beta)}{\left(z-z_{1}\right)\left(z-z_{2}\right)}
$$

$H_{c}(z)$ is a phase lead compensator:

$$
H_{c}(z)=1-\alpha z^{-1}
$$

$H_{F_{F_{-}} e}(z), H_{M F_{-} e 1}(z)$ and $H_{M F_{-} e 2}(z)$, shown in (5) (6) (7), are the electronic filters of the FF and the MF, respectively.

$H_{F F_{-} e}(z)=\frac{a_{1} \cdot\left[(z-1)^{2}+g \cdot k_{1} k_{2}\right](z-1)+a_{2} k_{1} \cdot(z-1)^{2}+a_{3} k_{1} k_{2} \cdot(z-1)+a_{4} k_{1} k_{2} k_{3}}{\left[(z-1)^{2}+g \cdot k_{1} k_{2}\right](z-1)}$

$H_{M F_{-} e l}(z)=\frac{a_{4} k_{3} \cdot\left[(z-1)^{2}+g \cdot k_{1} k_{2}\right]+a_{3} k_{2} k_{3} \cdot(z-1)+a_{2} k_{1} k_{2} k_{3}}{\left[(z-1)^{2}+g \cdot k_{1} k_{2}\right](z-1)}$

$$
H_{M F_{-} e 2}(z)=\frac{a_{1} k_{1} k_{2} k_{3}}{\left[(z-1)^{2}+g \cdot k_{1} k_{2}\right](z-1)}
$$

In order to make a comparison between the two architectures, a high performance accelerometer based on the bulk-machining technology and vacuum packaging, suffering from low Brown noise, is used for the system. Lumped parameters of the under-damped sensing element are listed in Table 1.

Table 1. Lumped parameters of the under-damped sensing element.

\begin{tabular}{|c|c|c|}
\hline Parameter & Value & Unit \\
\hline Mass & $7.34 \mathrm{e}-6$ & $\mathrm{~kg}$ \\
\hline $\begin{array}{c}\text { Damping } \\
\text { coefficient }\end{array}$ & $6 \mathrm{e}-3$ & $\mathrm{~N} / \mathrm{m} / \mathrm{s}$ \\
\hline Spring constant & 33.8 & $\mathrm{~N} / \mathrm{m}$ \\
\hline $\begin{array}{c}\text { Nominal electrode } \\
\text { gap }\end{array}$ & $2 \mathrm{e}-6$ & $\mathrm{~m}$ \\
\hline Area & $9 \mathrm{e}-6$ & $\mathrm{~m}^{2}$ \\
\hline Brown noise & 0.14 & $\mathrm{ug} / \mathrm{sqrt}(\mathrm{Hz})$ \\
\hline
\end{tabular}

A $62.5 \mathrm{~Hz}$ sinusoidal input signal with normalized amplitude of $-6 \mathrm{~dB}$ of the full scale is used to avoid overloading, and the simulation results of power spectral density (PSD) are presented in Figure 3. It is clear that both architectures achieve excellent noise shaping ability, suppressing the noise floor below $-170 \mathrm{~dB}$ within $250 \mathrm{~Hz}$ signal band. The estimated SNR values are $143.5 \mathrm{~dB}$ and $143.1 \mathrm{~dB}$ for the $\mathrm{FF}$ and the MF when the oversample rate (OSR) is 256 . 


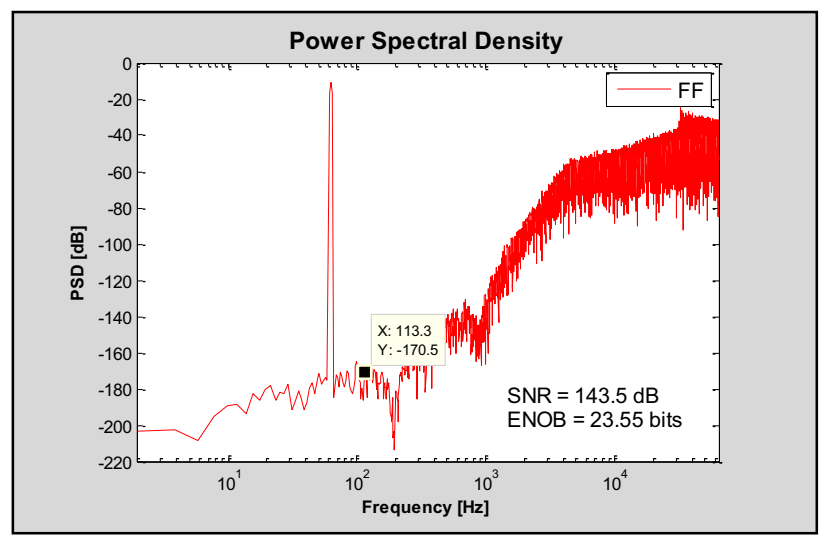

(a) FF

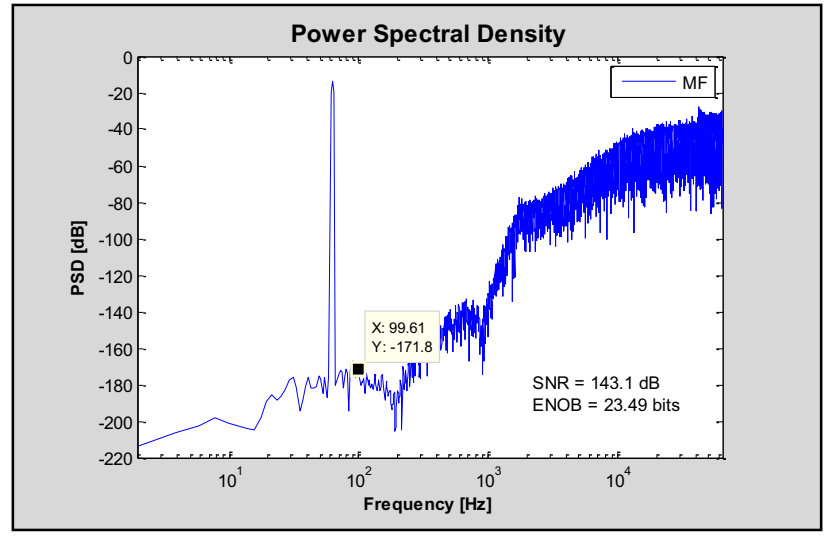

(b) MF

Figure 3. Noise shaping and SNR comparison for the FF and the MF.

\section{Architectural advantages disadvantages}

In all-electronic SDMs, the two architectures give similar performance. An electromechanical SDM, however, includes the sensor element as part of the loop filter. In this section, we will make a comparison between the FF and the MF architectures with regard to some important practical issues. The results given here will be a useful reference for the designers to decide which one is their better choice.

\subsection{Parameter sensitivity}

Limited to current fabrication technique, the sensing element parameters are often varied by $10 \%$ of their ideal values, which may break the system stability easily. As a result, the sensitivity of the two architectures to the sensor parameter variations derived from fabrication tolerances is a significant comparison criterion. These deviations contribute to a change in the dynamic characteristic of the sensing element, affecting the noiseshaping ability of the whole system.

In Figure 4, the three lumped model parameters of the MEMS accelerometer: proof mass ' $\mathrm{m}$ ', damping coefficient ' $b$ ', and spring constant ' $k$ ' are examined individually, to observe the parameter with the most critical effect. In this case, both of the two systems can be considered to be robust to parameter variation within the range of $\pm 10 \%$, since the SNR only suffers from within $2 \mathrm{~dB}$ degradation.

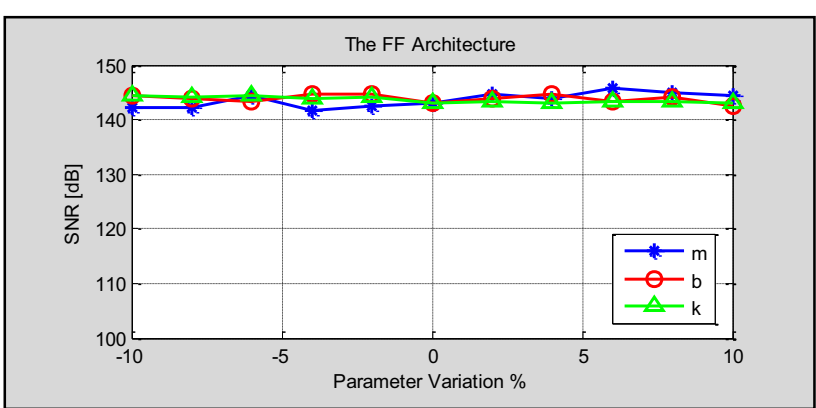

(a)FF

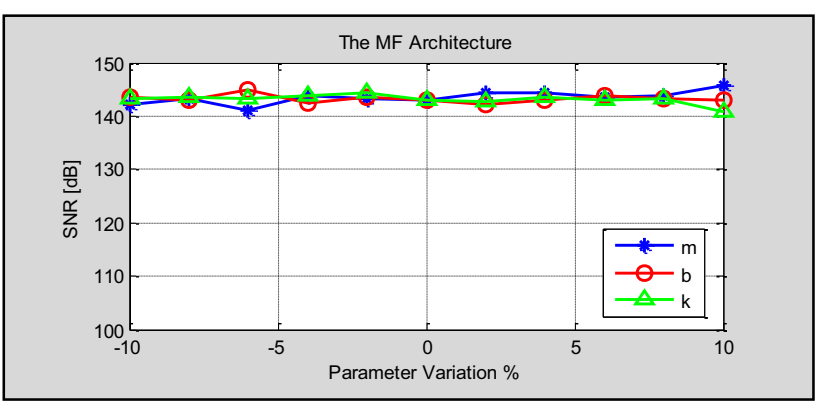

(b)MF

Figure 4. Parameter sensitivity analyse for the FF and the MF.

\subsection{Sensitivity to the loop gain}

Comparing equation (1) with (2), we can find that, in the FF, the mechanical element appears in series with the electrical filter and the quantizer. Since a 1-bit quantizer only outputs the sign of its input signal, this implies that any change in the loop gain independent of the frequency, such as Kfb, Kxv, will not affect the system stability. The $\mathrm{MF}$, on the other hand, has an additional purely electrical signal path, which bypasses the mechanical element, other than their common electromechanical signal path. It contributes poles of QNTF dependent on the values of the feedback coefficients as well as the parameters of the sensor. Since the feedback coefficients do not track the variation in the sensor transfer function, there is uncertainty in the position of the poles of QNTF.

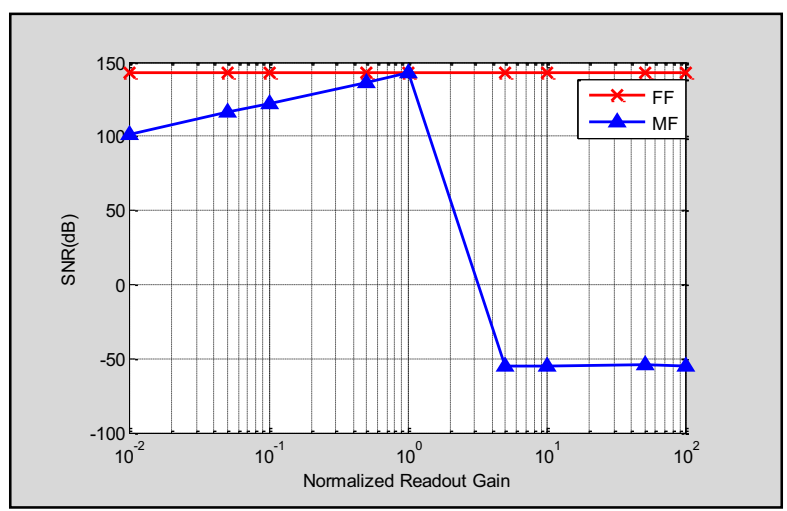

(a)Sensitivity to the readout gain 


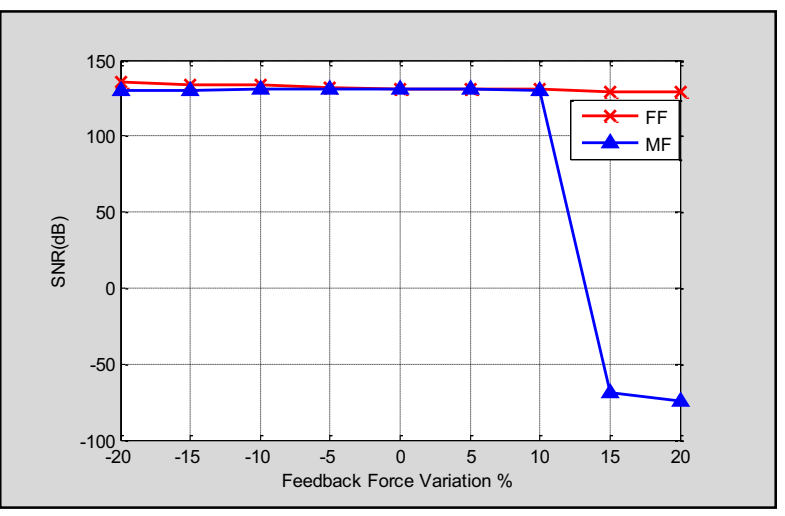

(b)Sensitivity to the feedback force

Figure 5. Sensitivity to the loop gain.

Figure 5 shows the changes of SNR in the FF and the MF with the normalized readout gain increasing from $10^{-}$ 2 to $10^{2}$ and the feedback force varying $\pm 20 \%$, respectively. Large changes in the loop gain has a negligible effect on the FF architecture performance, while the SNR of the MF degrades heavily, along with it decreases or increases. The tolerances on the feedback force means designers can improve the full range easily by applying a larger feedback voltage or longer feedback time. What's more, there is a possibility that the same FF architectures can be used with different sensors, while the MF is only designed for a special sensor.

\subsection{Nonlinearity in the feedback force}

In Figure 1 and Figure 2, the conversion of a feedback voltage to an electrostatic feedback force on the proof mass is assumed as a constant. Actually, it is nonlinear depending on the residual proof mass motion, and is given by:

$$
\begin{aligned}
K_{f b} & =\operatorname{sgn}\left(Y_{\text {out }}\right) \frac{K_{f b 0}}{\left[1+\operatorname{sgn}\left(Y_{\text {out }}\right)\left(x / d_{0}\right)\right]^{2}} \\
& =\operatorname{sgn}\left(Y_{\text {out }}\right) K_{f b 0}\left[1+\operatorname{sgn}\left(Y_{\text {out }}\right) \cdot 2\left(x / d_{0}\right)+3\left(x / d_{0}\right)^{2}+\operatorname{sgn}\left(Y_{\text {out }}\right) \cdot 4\left(x / d_{0}\right)^{3}+\cdots\right]
\end{aligned}
$$

where $K_{f b 0}=2 \varepsilon_{0} A V_{f b}^{2} / d_{0}^{2}$. Equation (8) indicates that the electrostatic feedback force has higher harmonic content, which will cause harmonic distortion, resulting in a reduction in the SNDR.

To investigate the feedback force nonlinear effect on an EMSDM performance, system level models with the nonlinear electrostatic feedback force were simulated for the FF and MF once time. Assuming a normalized amplitude of $-6 \mathrm{~dB}$ of the full scale input signal with a $62.5 \mathrm{~Hz}$ frequency, the maximum residual proof mass motion $\mathrm{x}$ of the FF and MF are about $1 \mathrm{~nm}$ and $17 \mathrm{~nm}$, respectively; this is shown in Figure 6. The spectrum of the output bitstream Yout is plotted in Figure 7. Since even harmonics are canceled due to the time-averaging positive or negative feedback pulse, there is only an obvious third harmonic distortion peak at three times of the input signal frequency (assumed to be $187.5 \mathrm{~Hz}$ ). The SNDR drops about $56 \mathrm{~dB}$ in a $250 \mathrm{~Hz}$ signal bandwidth in the MF, while it is too small that can be ignored in the FF.

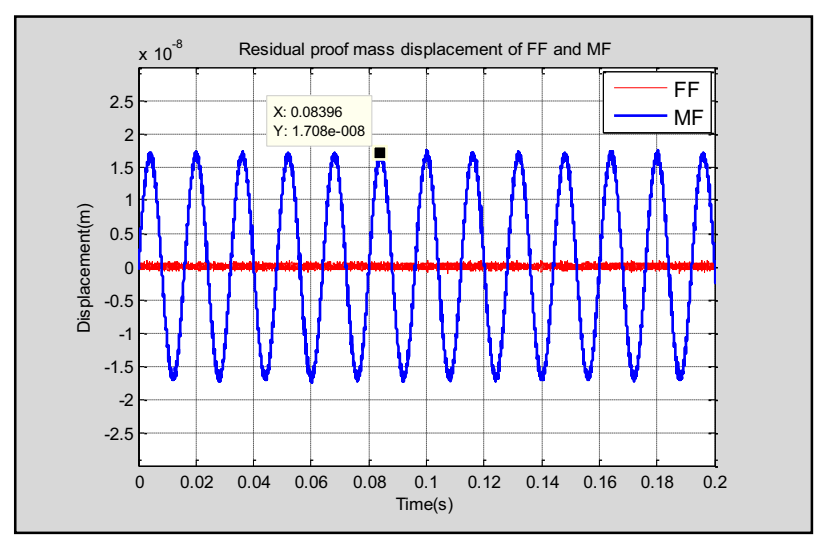

Figure 6. Residual proof mass displacement of a simulation run of the model in Figure 1 and Figure 2.

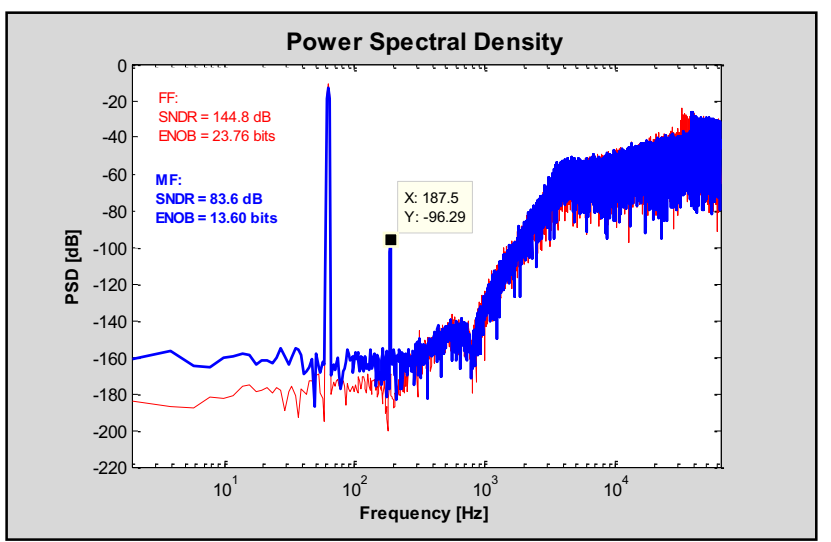

Figure 7. The output spectrum of a fifth-order EMSDM with a nonlinear force feedback.

This is not an accidental phenomenon. In purely electronic SDMs, the first integrator has much larger output amplitudes, which can be adjusted within a reasonable range by scaling the feedback coefficients a1, $\mathrm{k} 1$, than the FF. In EMSDMs, however, the gain of the sensing element cannot be changed, so what we can only do is changing the gain of $\mathrm{Kxv}$ to meet the stability requirement. Nevertheless, it is useless to narrow the displacement. Hence, we can conclude that, there exists more serious nonlinear, which means larger SNDR in the feedback force of the MF than the FF.

\section{Conclusions}

A comparative study was carried out between two $5^{\text {th }}$ order EMSDMs for a MEMS capacitive accelerometer: a feed-forward (FF) architecture and a multiple-feedback (MF) architecture. Both architectures achieved over 140dB SNR (Signal to Noise Ratio) and suppressed a quantization noise floor level below $-170 \mathrm{~dB}$ with a bandwidth of $250 \mathrm{~Hz}$. Besides, the investigation confirmed their immunity to the sensing element parameter variations due to manufacture errors. The FF can be used with different sensors directly with its superiority of large tolerance on the loop gain, while the MF can only be designed for a special sensor because its performance degrades seriously as the loop gain changes. The former has slight third harmonic distortion, since it controls the displacement of the proof mass much smaller 
than the latter. As such, we believe that the FF architecture will be more popular with designers.

\section{References}

1. Dong, Yufeng, M. Kraft, and C. Gollasch. "A High performance accelerometer with fifth-order SigmaDelta Modulator." Journal of Micromechanics \& Microengineering 15.7(2005)

2. Henrion, W., et al. "Wide dynamic range direct accelerometer." IEEE Solid-state Sensor \& Actuator Workshop IEEE. (1990)

3. Dong, Yufeng, M. Kraft, and W. Redman-White. "Higher Order Noise-Shaping Filters for HighPerformance Micromachined Accelerometers." Instrumentation \& Measurement IEEE Transactions on 56.5(2007)

4. Almutairi, Bader. Multi stage noise shaping (MASH) sigma delta modulator for capacitive MEMS inertial sensors. Diss. University of Southampton. ( 2015)

5. Y. Dong. Control systems for capacitive micromachined inertial sensors with high-order sigma-delta modulators. Diss. University of Southampton. (2006)
6. Zwahlen, P., et al. "Breakthrough in high performance inertial navigation grade Sigma-Delta MEMS accelerometer." Position Location and Navigation Symposium (PLANS), 2012 IEEE/ION IEEE. (2012)

7. Fu, Qiang, et al. "Design of high-performance fourth-order single-loop sigma-delta modulator for inertial sensor." Optoelectronics and Microelectronics (ICOM), 2012 International Conference on IEEE.( 2012)

8. Zhang Feng, X. Jin, and L. Wang. "Modeling and test result of closed-loop MEMS accelerometer with wide dynamic range." Microsystem Technologies . (2015)

9. Wilcock, Reuben, and M. Kraft. "Genetic Algorithm for the Design of Electro-Mechanical Sigma Delta Modulator MEMS Sensors." Sensors11.10 (2011)

10. Norsworth, S.N.; Schreier, R.; Temes, C. DeltaSigma Data Converters, Theory, Design, and Simulation; IEEE Press: Piscataway, NJ, USA. (1997)

11. Xuesong Jiang. Capacitive Position-Sensing Interface for Micromachined Inertial Sensors [D]. University of California at Berkeley. (2003) 